\title{
The "Invisible Hand" of Economic Markets Can Be Visualized through the Synergy Created by Division of Labor
}

\author{
Klaus Jaffé \\ Universidad Simón Bolívar, Caracas, Venezuela \\ Correspondence should be addressed to Klaus Jaffé; kjaffe@usb.ve
}

Received 6 March 2017; Revised 10 September 2017; Accepted 21 November 2017; Published 18 December 2017

Academic Editor: Pietro De Lellis

Copyright ( 2017 Klaus Jaffé. This is an open access article distributed under the Creative Commons Attribution License, which permits unrestricted use, distribution, and reproduction in any medium, provided the original work is properly cited.

\begin{abstract}
Inspired by Adam Smith and Friedrich Hayek, economists promoting free markets postulate the existence of invisible forces that drive economic growth. Simulations with Sociodynamica allowed the emergence of market forces in virtual economies, showing that the synergistic working of division of labor in complex settings favors a stable state where all actors benefit (winwin interaction). By visualizing the detailed dynamics underlying this phenomenon in a simple virtual economy, the elements underpinning the synergistic effect on economic output produced by the division of labor between agents could be dissected. These are heterogeneity or spatial or temporal heterogeneous environment and/or agents; complementary activities of agents, with divergent optimization options; and synchrony. Markets help synchronize agent's actions. The larger the contact horizon between participants of the market is, the more efficient the market forces act. These features allow for social processes that increase the information available and increase simultaneously the capacity of producing useful economic work, that is, synergy. This insight, although trivial if viewed a posteriori, improves our understanding of the source and nature of synergies in real economic markets and might render economic and natural sciences more consilient.
\end{abstract}

\section{Introduction}

The term synergy in science was used in neuromuscular physiology by Sherrington [1] when he described the integrative action of the nervous system. The concept was further developed as a process involved in self-organization by the theoretical physicist Haken [2] and the biologist Corning [3]. Exploring "Google Books Ngram Viewer" for the word "synergy," ranging the years 1800 to 2000 , shows that the use of the word synergy starts after the year 1900. In modern economics, the term is widely used. A Google Scholar search (9/8/1017) of the terms "synergy" and "economics" revealed 216000 results of "synergy" and "mergers" 37300; of "synergy" and "Adam Smith" 12700; of "synergy" and "invisible hand" 6610; of "synergy," "invisible hand," and "simulation" 1540. This last search showed the paper by Marris and Mueller [4], review of the literature entitled "The Corporation, Competition and the Invisible Hand" where the word synergy appears only in the title of one of the papers in the 5 pages of references. This was the work by Carter [5] entitled "In search of synergy: A structure-performance test." Here, Carter wrote that "the possibility of synergistic effects to diversification is recognized by most if not all observers." So evident is the importance of synergy to economists that few write about it. More recently, research on virtual artificial societies or computer simulations of social dynamics (see reviews in $[6,7]$ for example) have shown their worth in illuminating how the aggregate of various simple interactions might produce phase transitions and the emergence of novel properties of the system and even novel phenomena. These modern computer simulations, specifically agent based simulations, allow us to explore complex economic phenomena. Examples include the complexity of exchanges [8] and money dynamics and banking catastrophes [9]. However, agent based simulations have not been incorporated in mainstream economics [10], nor have they unveiled until now in detail the working of the invisible hand of the markets. Agent based simulations are a powerful tool in clarifying fundamental aspects of the working of complex economic phenomena. Its potential in visualizing fundamental concept in very simple economies will be explored here so as to avoid the limits "of ascertaining all the data whose utilization in the allocation of resources is the 
great merit of the market system" (Hayek 1911), and which are not easy to determine analytically in more complex economic settings.

Here, a deeper analysis of the working of synergy in market economies is presented, using experiments with computer simulations written in different languages (C\#, Visual Basic 6, and JavaScript) that improved the robustness of my previous findings and provided more details of the economic mechanisms that trigger synergy. As complex system science has shown with many examples (climate change, meteorology, cosmology, city dynamics, complex networks, etc.), the emergence of complex phenomena, such as synergy, is the product of interactions of many elements. Presenting a simple analytical expression of this process creates a false illusion of simplicity. Thus, analytical formalisms that give this false impression are avoided and the focus is placed on the details of the algorithms that allow the synergistic processes to emerge.

Smith in his book The Wealth of Nations [11] described the operation of the market as follows: "Every individual necessarily labours to render the annual revenue of the society as great as he can.... He, however, does not have the slightest intention of promoting the public interest or is aware that he is promoting it. He intends only his own gain and is led, as in many other cases, by an invisible hand that makes him promote a cause that does not form part of his intentions. This is not a disadvantage for society. By pursuing his own interest, he frequently promotes that of the society more efficiently than if his interest were the latter. I do not know of much good dispensed by those who strive to represent the common good. It is not from the benevolence of the butcher, the brewer, or the baker, that we can aspire to our dinner, but from their attention to their own interests." In another part of the book he writes "The greatest improvement in the productive powers of labor, and the greater part of the skill, dexterity, and judgment with which it is anywhere directed or applied, seem to have been the effects of the division of labor.... It is the great multiplication of the productions of all the different arts, in consequence of the division of labor, which occasions, in a well governed society, that universal opulence which extends itself to the lowest ranks of the people." These are beautiful descriptions of phenomena where the interactions at the individual level bring as a consequence dynamics significant only at the social level, without individual activity being conscious of it. It is a fascinating phenomenon but difficult to study using traditional experimental techniques.

The discovery of the invisible hand of the market is a major achievement of humankind. It recognizes the absence of centralized social cohesive forces and discovers forces of the market that explain our social dynamics. More detailed studies of the effects of division of labor have been published ([12], e.g.), but all failed to grasp analytically in ways acceptable to the natural sciences the emergence of synergies in economic markets due to division of labor. This inability to grasp numerically these phenomena has led some economists to conclude about the analytical intractability of all details in complex economies. Prominent among these thinkers is Hayek [13], who coined the term "Economic Calculus" when referring to this fundamental analytical limitation of economic analysis. He said [14] "[economics] has become too ambitious by applying standards of rigorousness... to the empirical science of economics where there are definite limits to what we can positively now; that we shall see more clearly what economics can do if we separate that logical groundwork - the economic calculus as I have called it - from its use in the empirical science of economics; and that, though this science is of great help in all-important issues of the choice of an economic order and of the general principles of economic policy, its power of specific prediction is inevitably limited - limited by the practical impossibility of ascertaining all the data - those very data whose utilization in the allocation of resources is the great merit of the market system."

In theory, the adaptation to dynamic fitness landscapes by biological populations of individuals with complexly interrelated gene assembles can be studied with an evolving multiplex network analysis where population evolves in a multilayered network with nonlinear complex interactions between the nodes of the different networks. Adaptive networks have been described for simple networks with linear interactions (see, e.g., [15]), However, the problem tackled here requires a dynamic multiplex network that produces adaptive evolution of a population of agents with a layer of networks representing the spatial environment where agents interact and which provides the selection forces that act on individuals, a second layer that mirrors the allelic composition of the genes in the genome of each agent and its intraindividual interactions, a third layer reflecting the interindividual interactions of the allele of all genes in the population, and layers that code for the nonlinear interactions of each allele in each gene locus. Only with these layers can we attempt to represent the agents and their reproductive interactions, the genetic modulation of individual fitness, kin selection, and inclusive fitness. Multilayered networks have shown to be unable, up to now, to explain cases as complex as that of the evolution of sex [16]. In contrast, agent based simulations provide an expedite route to study these phenomena. This rational is at the base for the choice of agent based simulations for studying the emergence of synergies in economics.

Simulations with the agent based simulation Sociodynamica [17] allow for exploring abstract virtual economies that are far simpler than real ones but already so complex that the experimenter may lose the integral view over the interactions between environment, agent behavior, pricing mechanisms, and environmental heterogeneity in the market. This might happen in simulations of economies where realistic price dynamics were included that showed that division of labor was the strongest predictor of successful economic performance [18]. However, it was not clear in these simulations, if this effect of division of labor was exerted through the price dynamics of the economy or through other means. In order to pinpoint the source of the synergies achieved by division of labor, the model was simplified until the emergent effect of division of labor disappeared. Stripping out effect of pricing on the market dynamics did not eliminate the effect of division of labor. This allowed us to follow in detail the features that make division of labor work, making the system amenable to analytical analysis. 


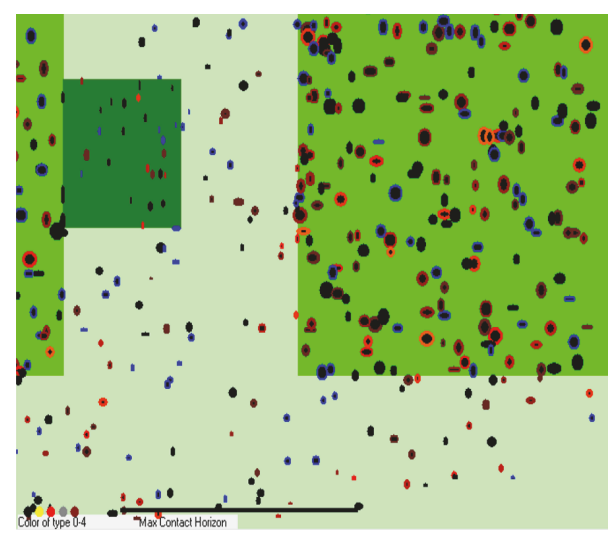

(a)

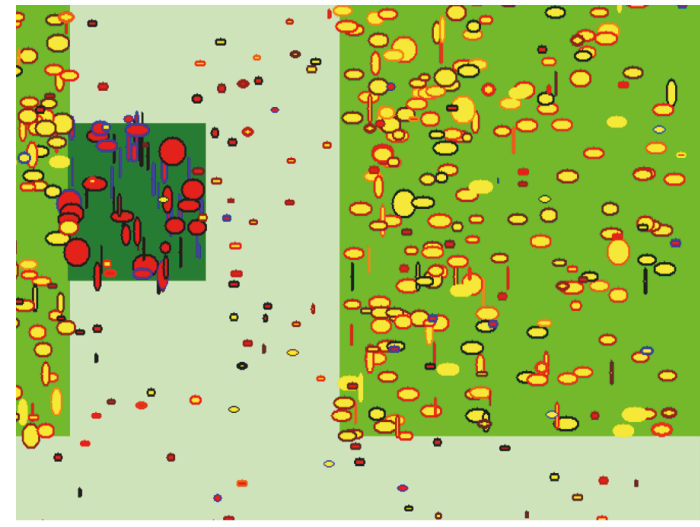

(b)

FIGURE 1: Representation of the landscape of two virtual economies. (a) shows a result from a simulation with free prices in an economy collecting food and minerals by omnipotent agents, whereas (b) shows the same but for agents specialized either in mining or farming.

\section{The Model}

The agent based computer simulation model Sociodynamica is a freely available agent based simulation model, first written in Visual Basic, and has previously been used to study the effect of altruism and altruistic punishment on aggregate wealth accumulation in artificial societies and to grasp the dynamics of complex markets (see [17-19] and references therein). These models are completely mechanical in nature, and individual incentives may emerge trough an evolutionary process that makes agents with the right combination of incentives or behaviors survive and those with the wrong combination to become eventually extinct. The features revealed by Sociodynamica are very similar and, in cases identical, to those revealed by Sugarscape, an agent based model developed independently by Axelrod [20] or the model developed by Axtell [8]. In all three cases, Walrasian solutions in which an auctioneer centrally computes prices cannot be made more efficient than the decentralized alternatives based on free and heterogeneous agents making these decisions. These results support the proposition of Adam Smith that markets are ruled by an invisible hand that coordinates the different kind of labor rendering markets efficient. Specifically, simulations in complex economic setting showing omnipotent agents performing all tasks produced less aggregate wealth than simulations where three different agents performed different tasks, such as farming, mining, and trading [18]. This counter-intuitive result was partly due to the fact that optimal prices and conditions for trade were different for each agent, depending on its spatial position in the virtual world. Omnipotent agents had to assume average solutions to balance their different tasks. Therefore, they never traded at optimal prices and optimal quantities according to their spatial position. Here the same model was used to run simplified scenarios, until the effects of prices dissipated, to reveal fundamental economic features that allow the emergence of synergies from division of labor.
The model simulates a virtual society of agents who farm and mine for foods and minerals, respectively, analogous to the model "Sugarscape" by Axelrod [21] and also trade their surplus according to different economic settings. The agents inhabit in a continuous flat two-dimensional toroidal world (see Figure 1) that was supplied with patches of agricultural land ("sugar" or "food") and separate nonoverlapping patches of mines ("spices" or minerals). Diverse agents were distributed at random on a fine-grained virtual landscape with resources. Simulations depended on the type of movement of agents, and, thus, to simplify interpretation of results, agents were simulated as immobile entities. Their individual utility function was defined by two resources. Each time step, any agent that happened to be located over one of these resources acquired a unit of the corresponding resource, accumulating its wealth, either as sugar or food $\left(G_{1}\right)$ and/or as spices or minerals $\left(G_{2}\right)$. Agents spend a fixed amount of each resource in order to survive, consuming each of them at a basal constant rate (default value was set to 0.1 units of the corresponding resource at each time step). Both resources were consumed and metabolized similarly, but food was 3 times more abundant than minerals (the size of the patch for minerals was set to $100 \times 100$ pixels and for food was $300 \times 300$ pixels). Each patch remained in the same place during each simulation run and the resources inside them were replenished continuously. Agents perished when they exhausted any of the two resources. Success in gathering and trading resources was defined by variables that produced behaviors that made them unable to compete successfully for resources. These variables included type of movement, spatial positioning, price thresholds for selling each of the resources, price threshold for buying the resources, and type of agent. During the simulation, natural selection weeded out unsuccessful combinations of these variables. The total population of agents was maintained constant by creating the required amount of new agent necessary, each with randomly assigned initial parameters. Initial parameters were the type of agent, the random spatial position, and the initial amount 
of money used to start trading resources (the default initial value was set to 10 units of money). The amount of money for each agent varied according to its trade balances. Agents gain money when selling food and/or minerals and lose money when buying them.

Agents traded the resources they possessed with other agents. In order to trade, they had to find a partner with the desired resource, and they had to have agreement over prices. The trade could be among any agent in a population of omnipotent agents without "division of labor." When simulating division of labor, agents specialized in collecting food or collecting minerals or collecting neither but engaging only in trade. Here, agents were subdivided into three categories. Farmers which specialized in collecting only resource 1; Miners which extracted only resource 2; Traders specialized in trading minerals for food when encountering a farmer, and food for minerals when encountering a miner. Food collectors traded only with mineral collectors and Traders, mineral collectors traded only with food collectors and Traders, and Traders could interchange resources with all types of agents. Trades were allowed only between agents spaced at a distance not larger than the "contact horizon" of the trading agent. Each time step, all buyers searched for potential sellers of the required good by contacting randomly up to 10 agents in the area defined by this contact horizon. If finding a seller with the wanted goods at or below the price defined by the buyer, a trade was executed using the price of the seller. Trades were limited to the amount of money available to the buyer and the amount of goods possessed by the seller, unless credit was simulated. Variation of this contact horizon allowed simulating different levels of globalization or integration of economic agents. The effect of the degree of globalization (or the size of the market) on the economy can thus be measured quantitatively, a feature that is not possible with real economies (but see [22]).

Prices were initially assigned to each agent for each resource at random from a range of values defined by the experimenter and then varied according to supply and demand as experienced by each individual agent. That is, at the end of every time step, after finishing a tournament of trades in the market, each selling agent attempting to sell parts of its excess of resource that could not find a willing buyer because of the price it asked for reduced its reference price by an unit. And each buyer that could not find a seller willing to sell the desired resource at the desired price increased its reference price for that resource. In this way, each agent maximized its self-interest by selling each resource at the maximum price possible and buying at the lowest.

Various processes were simulated. A first process of the simulation was the balance between income (I) of resources (r) and their consumption (C). For survival, agents were required to conform to

$$
\mathrm{Ir}>\mathrm{Cr} \text {. }
$$

Income can be either by direct gathering $(\mathrm{G})$ or by trade $(\mathrm{T})$

$$
\mathrm{Ir}=\mathrm{Gr}+\mathrm{Tr} .
$$

Here each agent has to balance two resources in order to survive. A utility function $(\mathrm{U})$ is simulated so that $\mathrm{U}$ remained positive for the agent's survival and

$$
\mathrm{Ur}=(\mathrm{Gr}+\mathrm{Tr})-\mathrm{Cr} .
$$

A second process of the simulation was the dynamics of traded resources. These resources can increase or decrease, according to the balance of resources bought (B) and sold (S)

$$
\mathrm{Tr}=\mathrm{Br}-\mathrm{Sr} .
$$

The amount of resources bought and sold depends on the availability of money $(\mathrm{M})$ and the price $(\mathrm{P})$ paid for the resource by the agent (a)

$$
\begin{aligned}
& \mathrm{Br}=\frac{\mathrm{M}_{\mathrm{a}}}{\mathrm{Pr}_{\mathrm{a}}}, \\
& \mathrm{Sr}=\frac{\mathrm{M}_{\mathrm{a}}}{\mathrm{Pr}_{\mathrm{a}}} .
\end{aligned}
$$

The amount of money of agent (a) depends on the amount spend buying (Mbr) and the amount gained selling (Msr) resources

$$
\mathrm{M}_{\mathrm{a}}=\mathrm{M}_{\mathrm{a}} 0+\mathrm{Msr}-\mathrm{Mbr},
$$

where $M_{a} 0$ stands for the initial amount of money supplied to each new agent $j$.

Ur was calculated every time step for the population of agents (a) so that the total accumulated of wealth for each resource $(\mathrm{Wr})$

$$
\mathrm{Wr}=\Sigma_{\mathrm{a}} \mathrm{Ur} .
$$

Agents with $U_{1}<=0$ or $U_{2}<=0$ were eliminated and substituted by new ones with default properties, as an analogy of broken companies that are replaced by new start-ups.

Here the focus is on the age of the agents as the most relevant variable for assessing the benevolence of an economic system. The average age is a measure of the probability of survival of individuals in the population, but other measures are possible [18]. The aim was to pinpoint the features that allowed the emergence of the synergies of the market due to division of labor. Simulations of virtual worlds with one type of agents (omnipotent agents), two types (Farmers + Miners), and 3 types (Farmers + Miners + Traders) allowed determining the effects of increasing complexity of labor structure. Simulation with homogeneous and heterogeneous distribution of resources allowed assessing the effect of the environmental complexity, and simulations with different contact radius provided insights to the importance of synchronization between trading agents. Synchronization here means allowing the meeting of buyers and Traders.

A longer and more detailed description of the simulations is provided in Jaffe [18] and the detailed program in Visual Basic is available in the help feature of the program.

Sociodynamica has been rewritten in C\# and JavaScript. Simulations with these versions can be run with parameters chosen at will by downloading Sociodynamica. The Visual 
TABLE 1: Average age accumulated by agents after 200 time steps in simulations exploring the effect of fixed or free prices, with omnipotent agents or with agent dividing labor. Maximum contact radius of agents was 200 pixels. Each of the data is the mean of 100 simulation runs.

\begin{tabular}{lccr}
\hline & Omnipotent agents & $\begin{array}{c}\text { Division of labor 2 } \\
\text { Farmers + Miners }\end{array}$ & $\begin{array}{c}\text { Division of labor 3 } \\
\text { Farmers + Miners + Traders }\end{array}$ \\
\hline Fixed prices & $11.2 \pm 0.71$ & $86.9 \pm 3.5$ & $69.2 \pm 3.6$ \\
Free prices & $11.3 \pm 0.8$ & $87.7 \pm 3.2$ & $69.1 \pm 3.9$ \\
\hline
\end{tabular}

Basic 6 version is at http://atta.labb.usb.ve/Klaus/Programas .htm and the JavaScript Unity version at http://bcv.cee.usb.ve/ juegos/intro_en.html, where the C\# version motors the Unity version. Default values for Sociodynamica used here were contact horizon for transaction $=200$. For both resources, Initial prices $=3$ units and Reserve units not traded $=$ 1. Amount of resources metabolized per time step $=0.1$. Amount of resources collected per time by agent $=2$. Number of agents $=500$; maximum number of trades per time step $=10$. More details are given in Appendix. Videos of the simulations run for the present paper are available at http://atta.labb.usb.ve/Klaus/EC/ECVideos.html.

A user friendly game version of Sociodynamica is available at http://www.bcv.cee.usb.ve/juegos/intro_en.html.

\section{Results}

Figure 1 shows two examples of the output of simulations with free prices after 200 time steps. Figure 1(a) shows the virtual world when simulating omnipotent agents; Figure 1(b) reflects the outcome when division of labor (Farmers + Miners) was included in the simulations. The figures reflect the effects on the economic dynamics of introducing division of labor. In the figure with omnipotent agents, agents over mineral fields are smaller (have less wealth) than those over fields with food. In contrast, the figure from simulations including division of labor showed that Miners over field of minerals were very wealthy, and so where many farmers over field with food. The price agents were willing to pay for minerals was higher among farmers (red borders in Figure 1(b)) and lower among Miners (blue borders in Figure 1(b)) when division of labor was simulated. In the case of the omnipotent agents, prices agents were willing to pay for minerals or food seemed to be randomly distributed (mix of colors in borders of agents in Figure 1(a)). This example reveals that omnipotent agents made suboptimal trading decisions. They sold the resource they had accumulated in more abundance to any other agent willing to take it, whereas specialized agents (Figure 1(b)) traded only the resource they were collecting; that is, Miners only sold minerals to Farmers and Farmers sold only food to Miners. The trading patterns of omnipotent agents produced less wealth in the long term than that of specialized agents, even though the cognitive complexity of the algorithm omnipotent agents used was more complex.

(i) Bright green field is covered with "food"; darker green field is covered with "Minerals"; the lightest green is devoid of resources.

(ii) Each agent is depicted as a colored sphere. The color of the body of the sphere describes the type
TABLE 2: Average age accumulated by agents after 200 time steps in simulations exploring the effect of homogeneous economic environments. Maximum contact radius of agents was 200 pixels. Each of the data is the mean of 100 simulation runs.

\begin{tabular}{lcc}
\hline $\begin{array}{l}\text { Omnipotent } \\
\text { agents }\end{array}$ & $\begin{array}{c}\text { Division of labor 2 } \\
\text { Farmer + Miner }\end{array}$ & $\begin{array}{c}\text { Division of labor 3 } \\
\text { Farmer + Miner + Trader }\end{array}$ \\
\hline $197 \pm 4$ & $198 \pm 4$ & $176 \pm 7$ \\
\hline
\end{tabular}

of agent: Farmers are yellow, Miners are red, and omnipotent agents are black. The width of the bubble is proportional to the amount of food and minerals accumulated by the agent and the height by the amount of money the agent possesses.

(iii) The thickness of the border of the sphere is proportional to the perceived cost of living calculated as Food Price + Mineral Price and the color of the border ranges from blue to yellow. The more red or even yellow the higher the ratio between the Food price and Mineral price. Agents with red and yellow borders pay more for minerals, whereas those with blue or black borders pay less for minerals compared to what they are willing to pay for food.

As multivaried complex data is difficult to visualize, as the attempt in Figure 1 shows, data will be presented also in a more classical format.

The difference between the two simulations shown in Figure 1 is presented quantitatively in Table 1. As intended in this simplified model, the effect of centralized or decentralized pricing was stripped out, so that this feature did not affect results. Agents in simulations with 2 types of agents (Farmers and Miners) performed much better economically, achieving much longer life-spans in average than agents in simulations with only one type of agents (omnipotent agents). Increasing the complexity of the division of labor by including Traders, that is, agent that did not collect resources but only bought and sold them, using the same algorithm as that of omnipotent agents, did not further increase the efficiency of the resulting economy.

Eliminating heterogeneity in the environment eliminated the advantage of division of labor (Table 2). Simulations where fields of minerals overlapped in all its extension with field of food produced quantitative results that were identical between virtual economies of omnipotent agents and economies with division of labor with two type of agents ( $p>0.98$ for rejection of null hypothesis using Student's $t$ test). 
TABLE 3: As in Table 2, where agent types 1, 2, and 3 stands for omnipotent, divisions of labor 2 and 3. Cr indicates the presence of specialized traders dispensing credit.

\begin{tabular}{lccc}
\hline Price & Agent types & $\begin{array}{c}\text { Average } \\
\text { age }\end{array}$ & $\begin{array}{c}\text { Total goods } \\
\text { accumulated }\end{array}$ \\
\hline \multirow{5}{*}{ Variable } & 1 & 11 & 8052 \\
& 3 & 102 & 74150 \\
& $3 \mathrm{Cr}$ & 93 & 59184 \\
Constant & 1 & 101 & 45890 \\
\hline \multirow{6}{*}{} & 3 & 9 & 10252 \\
& $3 \mathrm{Cr}$ & 85 & 95140 \\
& & 87 & 63253 \\
\hline
\end{tabular}

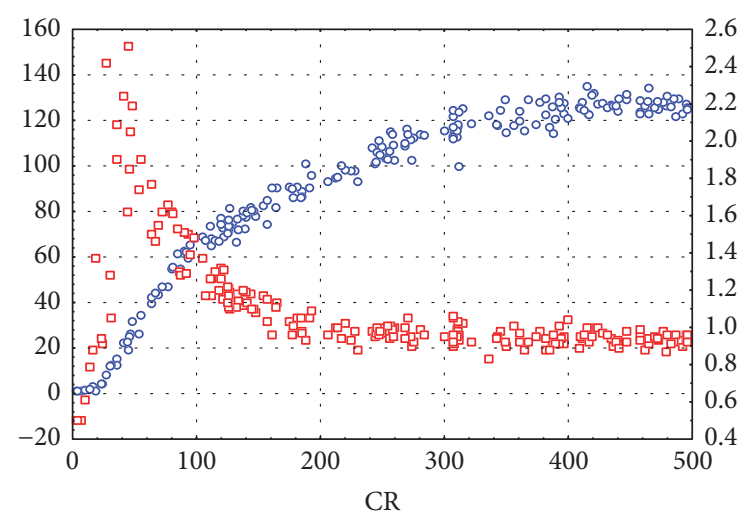

- Age-mean (L)

- $\operatorname{FPr}-\operatorname{mean}(\mathrm{R})$

FIGURE 2: Effect of the contact radius on the economic performance of the virtual economy, measured by the average age (blue circles, left scale) and average price of food red squares, right scale) accumulated by agents after 200 time steps in simulations with free prices and with division of labor.

Results show that the division of labor, in order to work properly, required an adequate coordination of actions with synergy between the agents. For example, omnipotent agents sold whatever resource they had, independently of what they collected, whereas Farmers and Miners only sold the product they collected and bought the one they did not collect. Including Traders in simulations introduce noise in the economy unless they had a specific function besides trade, such as providing credit, which benefited society in economies where prices could be adjusted through demand (variable prices) as shown in Table 3.

Other features also increased the possibility of improving the economy. In Figure 2 the effect of an improved ability to trade is shown. Here, the maximum contact radius determining the distance at which potential Traders could be spatially separated was varied. As expected, results show that, at greater maximum contact radius, the economy performed better. This trend, however, was not linear. Prices had a very strong nonlinear relationship with the contact radius.
This relationship strongly depended on the topology of the resource distribution simulated, as was shown before [18].

\section{Conclusions}

The experiments presented here showed that the same results are obtained with different software written in different computer language. This makes it unlikely that the result are due to coding artifacts rather than the logic of the causal network explored. The results presented here identified features that are indispensable for making the emergence of synergies, due to division of labor, possible. These are as follows:

(1) Heterogeneity: spatial or temporal heterogeneous environment and/or agents.

(2) Complementarity: complementary activities of agents, with divergent optimization options.

(3) Synchrony: markets help synchronize agent's actions. The larger the contact horizon between participants of the market is, the more efficient the market forces act.

These features allow the social system to decrease its entropy, increasing the information managed by the system, and at the same time increase the economic work performed. These two features have been proposed to thermodynamically characterize a synergistic process. Thus, economic activity can be meaningfully characterized using thermodynamic concepts providing a way to quantify synergistic processes [23].

The results are relevant to empirical findings in real economies. For example, the first feature is interesting in that it recognizes the fact that the complexity of labor structure mirrors the complexity of the economic environment where it works, and vice versa. This feature is a consequence of economic activity being molded by the environment to which it has to adapt. In human societies economic wealth of a country and economic complexity are linked very strongly [24]. This suggests that economic complexity, of course, can only be achieved with complex division of labor. This requires increased availability of information. This last requirement triggered the search for this relationship in real economies (Jaffe 2013), showing that management of scientific information is more closely related to economic output of a society than the complexity as measured by Hausmann and Hidalgo [24].

The second feature is illuminating as it allows predicting when division of labor might improve economic activity and when not. Division of labor might prevent inefficient trades and/or might make more efficient trades possible. Tasks, which converge in skills, might not require specializations, whereas tasks that require very different types of skills will benefit more of division of labor. It can be argued that more intelligent agents might be capable of performing several tasks and, thus, rather that division of labor, economies benefit from more complex or intelligent agents. This might be true but complexity and intelligence have their costs and might be suboptimal if different simple agents can 
handle the problems with higher efficiency. This seems to be the case in the social evolution of ants, where a negative correlation between brain development of individuals and social complexity was evidenced [25].

The third feature is possibly the more difficult to manage in real situations. Experience shows that on-time synchronized productive chains allow specialization of tasks to be more productive overall, whereas low division of labor is more tolerant to inefficient supply chains. These features explain many a difference between highly developed economies and ones with incipient industrialization and poor services. Empirical studies showing how heterogeneity in a network can create heterogeneity of behavior [26] exist. This is one of the ways social systems might achieve synergistic synchronization of different activities. More research in this area is urgently needed in order to explain differences in economic development between countries [27].

The identification of these 3 features as fundamental in allowing division of labor to elicit economic synergies might seem trivial. But browsing the literature, a great number of reasons have been postulated to explain the synergies created by economic markets. The problem seems far from solved (see [28]). Even if found to be trivial a posteriori, the simulations helped to identify the relevant features and discard superfluous ones. Bowles [29], for example, defined the invisible marker mechanism as a Nash equilibrium and its Pareto optimal, where the self-interest of each actor yields an outcome that maximizes the wellbeing of each. Here more details of how this can be achieved are presented. The three features unveiled here seem to be very general, relevant to system dynamics, biology, human society, and real economies. Possible empirically falsifiable predictions based on these tree features are as follows:

(1) Division of labor should be more developed in societies that exploit a greater variety of resources [24].

(2) Better communication and economic instruments are provided by more sophisticated financial systems which in turn provide better opportunities for synergies between economic actors in more complex economies. Novel communication technology, such as the Internet, can also broaden the contact radius of economic agents improving synchrony [30].

(3) More division of labor leads to more incompatibilities between skills required to perform them and thus to more diverse specialized education [31].

(4) Larger contact horizons or more globalization improves the synergies unleashed by division of labor as they broaden the scope for more diverse interactions between economic agents

Though new research purposefully designed to answer these questions should be designed. Division of labor is related to synergies associated with fundamental aspects of social systems in biology [32]: In human society, economic synergy and division of labor have important relationships. Research showed that more complex economies requiring more division of labor accumulate more wealth and produce higher economic growth [24]. Empirical data showed that an even better predictor than economic complexity for future economic growth in developing countries is the scientific knowledge estimated by the amount of academic activity. Even more striking, the type of division of intellectual activity in a country is a much better predictor than the total complexity or the absolute amount of academic research performed [33]. That is, division of academic labor that prioritizes basic natural sciences over applied sciences and social sciences is much more efficient in producing future economic growth. These results, in the light of the findings of the present simulations, show that much remains to be learned about the quality and quantity of division of labor and its effect on economic activity. More interdisciplinary research is needed to improve our understanding of this very fundamental phenomenon.

This exercise shows that computer simulation of simple economic agents can generate nonlinear dynamics that resembles real life features of known economic system. Simulations of very complex systems produce complex results that may become intractable even with sophisticated statistical analysis. Here this limitation is avoided by focusing on very specific and fundamental problems. The simulations presented revealed fundamental features that allow division of labor to create economic synergies. This feat is impossible in complex real situations, but the insights gained in simple systems help in understanding synergies in more complex ones. Simulation models, besides having a potential in experimental economic research, are a fantastic tool to make complex phenomena visible to human understanding and thus should have a potential, if properly adapted for that purpose, in didactic games for teaching economics at all levels of educational and academic specialization. Science learned through smart games based on simulations, such as made available at http://bcv.cee.usb.ve/juegos/intro_en.html, might reduce self-serving cognitive biases among lay people, professional practitioners, and decision makers, improving the rationality of our society and thus, hopefully, its economic performance.

\section{Appendix}

Sociodynamica creates a virtual society where agents exploit and compete for resources and share resource 1 among them, according to the settings defined by the internal parameters and the external parameters. The agents may acquire renewable and nonrenewable resources through work; they may accumulate those resources and commercialize them. At the same time, agent may acquire resources through commerce.

\section{Global Parameters}

POP: number of agents (no)

$T A R$ : aggregate total wealth accumulated by all agents

Simulation Logic

Each Time Step

Do simulation loop

Matrix:

Eliminate variable types previously defined Eliminate agents with wealth $=0$ (par 11) Increment age agent $(i, 7)=\operatorname{agent}(i, 7)+1$ 
Use of resource 1 and 2 agent(i, 11)- BRCl; agent $(i, 12)$ - BRC2

Assessment of GDP GDP $=$ GDP + agent $(i, 11)$ Show and Plot

Plot Shows the agents according to their total resources (Food + Money) indicated as the sqr of the diameter.

The vertical size of the agent is proportional to the total wealth of the agent's money.

The color of the bubble depends of the type of agent as indicated at the left bottom of the screen.

The thickness of the border is proportional to the perceived cost of living (Prize for food + Price for minerals).

The color of the border gives the ratio MinPrice/ FoodPrice. Yellow and then red give the highest ratios. Blue borders indicate that food prices are higher than mineral prices.

Black bar at the bottom indicates a length of 100 pixel.

\section{Internal Parameters}

General Parameters (number in parenthesis indicates the column in the master matrix)

\section{$X(0)$ : Spatial dimension 1}

$Y$ (1): Spatial dimension 2

CRa (2): Contact Radius or Contact Horizon, maximum distance at which interchange between agents may occur (altruistic interchange for example)

TMo (4): Type of Movement; type of spatial displacement: no movement

\section{Characteristics of Agents}

Age (7): Age; Age of agent

WT (10): Wealth-Money; Total capital in liquid money

WFo (11): Wealth-food; Amount of resource 1 (Renewable). If $\mathrm{WFo}(\mathrm{i})=0$ then agent $\mathrm{i}$ starves ( $\mathrm{i}$ is eliminated)

WCo (12): Wealth-Commodity; Amount of resource 2 (Minerals or Nonrenewable resource)

Dept (13): Dept; Accumulated Dept

Well (19): Wellbeing; Amount of resources $3=\mathrm{r} 1 * \mathrm{r} 2$ * Gain

TAg (20): Type of Agent; Specialization or task of agent ( 0 omnipotent: exploits resources 1 and 2, 1 Farmer: exploits only resources 1, 2 Miners: exploits only resources 2, 3 Trader: does not collect but trades and provides credit, that is, creates money)

\section{External Parameters}

General

Initial Nr. of Agents (ino)

Optimum Population Size (ops). Maximum number of agents aimed at through ssconst
Simulation Scenario. Production of new agents (ssconst):

0: New agents are created each time step, until the number indicated by ops is achieved. New agents are assigned internal parameters at random

Proportion Culled (PC). The proportion of agents killed randomly when population in excess of ops.

A danger other than starvation is random selection. Large values increase random selection; large WCo reduces this.

Fitness Function. Agents, in order to continue in the virtual word, had to satisfy each time step the rule: $100 *$ $\mathrm{Rnd} /($ amount of resource 2$) /($ mean wealth of resource 2$)<$ $1000 *$ Rnd.

Dangers. There is a probability of being eliminated in random selection events

Resources 1 and 2

Resource 1 (Provides WFo) and 2 (Provides WCo)

Number of Patches of Resource (RNR). It is number of resource patches,

Size of Patch of Resource (SNR). It is maximum sizes of each patch (but see Mutation).

Degradation of Resource due to Consumption (DNR). It is amount lost due to consumption (RD)

Distribution Pattern of Resource (DPR). Resource is distributed:

(1) Fixed size, randomly distributed

(2) Fixed size, centered

Else Random size, randomly distributed

Basal Rate of Metabolism (BRC). It is amount of resource passively used-up (b).

Efficiency of Consumption (EfC). It is amount of resource assimilated.

When EfC > 10 then productivity is simulated: Agent collects $=(\mathrm{EfC}-10) *$ price $($ either for resource 1 and/or 2$)$.

Frequency of Change in Distribution (FCh). Frequency in $t$ steps distribution changes.

Consumption of Resource. Rate of exploitation: resource $=$ resource - DNR

BRC: Wearing or passive use of resource agent- BRC

Resource 1 can be modeled as a renewable resource (agriculture, for example),

whereas resource 2 as a nonrenewable resource (mining, for example),

by assigning $\mathrm{DNR}=0$ and $\mathrm{DNR}=1$ respectively

Food Reserve (FR). It is minimum amount of food needed for agent to engage in transactions of any kind. 
Min Food for Reproduction (MFR). It is the amount of food that needs to be accumulated before reproduction can start when Simulation Scenario is 1 or 4 .

Type of Economy (EconoT)

0 : No Barter nor any other interactions except taxes

1: Barter: with no money

$>1$ : Money as Species. If Price adjust $=0$ then Fixed prices

If Price Adjust $>0$ the Prices are determined by demand: agents not selling decrease price by one unit; agents not finding seller from which to buy increase price by one unit.

3: + Financial: Traders lend money.

4: + Agents finishing a successful sale, increase price by one unit

5: + Successful buyers decrease their future asking price by one unit

6: + As in EconoT 4 and 5

$7:+$ As in $2,3,4$ and 5

8: As in 2 and 3

10: Agents pay Taxes: Taxes collected are increased synergistically by Taxpool * SSTax prior to their distribution

Food Price. It is in integer units.

Mineral Price. Price of commodities is in integer units.

Price Adjustment. Units prices are reduced or increase when trade fails. $0=$ fixed prices.

\section{Conflicts of Interest}

The author declares that there are no conflicts of interest regarding the publication of this paper.

\section{Acknowledgments}

Thanks are due to Stephen Davies and Juan Carlos Correa for helpful comments on previous versions of the manuscript.

\section{References}

[1] C. S. Sherrington, The integrative action of the nervous system, CUP Archive, 1910.

[2] H. Haken, Instability hierarchies of self-organizing systems and devices. Advanced synergetics, Springer Science \& Business Media, 2012.

[3] P. Corning, Holistic Darwinism: Synergy, cybernetics, and the bioeconomics of evolution, University of Chicago Press, 2010.

[4] R. Marris and D. C. Mueller, "The Corporation, Competition, and the Invisible Hand," Journal of Economic Literature, vol. 18, pp. 32-63, 1980.

[5] J. R. Carter, "In Search of Synergy: A Structure-Performance Test," The Review of Economics and Statistics, vol. 59, no. 3, pp. 279-289, 1977.
[6] L. Tesfatsion, "Chapter 16 Agent-Based Computational Economics: A Constructive Approach to Economic Theory," Handbook of Computational Economics, vol. 2, pp. 831-880, 2006.

[7] N. R. Magliocca, D. G. Brown, and E. C. Ellis, "Cross-site comparison of land-use decision-making and its consequences across land systems with a generalized agent-based model," PLoS ONE, vol. 9, no. 1, Article ID e86179, 2014.

[8] R. Axtell, “The complexity of exchange," Economic Journal, vol. 115, no. 504, pp. F193-F210, 2005.

[9] C. D. Brummitt, R. Sethi, and D. J. Watts, "Inside money, procyclical leverage, and banking catastrophes," PLOS ONE, vol. 9, no. 8, Article ID e104219, 2014.

[10] R. Leombruni and M. Richiardi, "Why are economists sceptical about agent-based simulations?" Physica A: Statistical Mechanics and its Applications, vol. 355, no. 1, pp. 103-109, 2005.

[11] A. Smith, The wealth of nations, 1776.

[12] G. S. Becker and K. M. Murphy, "The Division of Labor, Coordination Costs, and Knowledge," in Human Capital: A Theoretical and Empirical Analysis with Special Reference to Education, G. S. Becker, Ed., pp. 299-322, 3rd edition, 1994.

[13] F. A. Hayek, Individualism and Economic Order, Rutledge Press, 1948.

[14] F. A. Hayek, Closing sentence of his final Virginia lecture, as reported by Bruce Caldwell (Duke University), 1961 in a paper prepared for a conference on Friedrich Hayek and the Liberal Tradition, University of Richmond, 2013.

[15] W. Yu, P. DeLellis, G. Chen, M. di Bernardo, and J. Kurths, "Distributed adaptive control of synchronization in complex networks," Institute of Electrical and Electronics Engineers Transactions on Automatic Control, vol. 57, no. 8, pp. 2153-2158, 2012.

[16] F. Schweitzer and Garas. A., Eds., Interconnected networks, Springer, 2016.

[17] K. Jaffe, "An economic analysis of altruism: Who benefits from altruistic acts?" JASSS, vol. 5, no. 3, 2002.

[18] K. Jaffe, "Visualizing the Invisible Hand of Markets: Simulating Complex Dynamic Economic Interactions," Intelligent Systems in Accounting, Finance and Management, vol. 22, no. 2, pp. 115132, 2015.

[19] K. Jaffe, "Evolution of shame as an adaptation to social punishment and its contribution to social cohesiveness," Complexity, vol. 14, no. 2, pp. 46-52, 2008.

[20] R. Axelrod, "Advancing the Art of Simulation in the Social Sciences," in Simulating Social Phenomena, vol. 456 of Lecture Notes in Economics and Mathematical Systems, pp. 21-40, Springer, Berlin, Germany, 1997.

[21] R. Axelrod, The Evolution of Cooperation, Basic Books, New York, NY, USA, 1984.

[22] N. F. Campos, F. Coricelli, and L. Moretti, "Economic Growth and Political Integration: Estimating the Benefits from Membership in the European Union Using the Synthetic Counterfactuals Method," IZA Discussion Paper No. 8162, 2014.

[23] K. Jaffe and G. Febres, "Defining synergy thermodynamically using quantitative measurements of entropy and free energy," Complexity, vol. 21, no. S2, pp. 235-242, 2016.

[24] R. Hausmann and C. A. Hidalgo, The atlas of economic complexity: Mapping paths to prosperity, MIT Press, 2014.

[25] K. Jaffe and E. Perez, "Comparative study of brain morphology in ants," Brain, Behavior and Evolution, vol. 33, no. 1, pp. 25-33, 1989. 
[26] A. Godoy, P. Tabacof, and F. J. Von Zuben, "The role of the interaction network in the emergence of diversity of behavior," PLoS ONE, vol. 12, no. 2, Article ID e0172073, 2017.

[27] J. C. Correa and K. Jaffe, "Corruption and Wealth: Unveiling a national prosperity syndrome in Europe," Journal of Economics and Development Studies, vol. 3, no. 3, pp. 43-59, 2015.

[28] S. Kochugovindan and N. J. Vriend, "Is the study of complex adaptive systems going to solve the mystery of Adam Smith's 'invisible hand'?" Independent Review, vol. 3, no. 1, pp. 53-66, 1998.

[29] S. Bowles, Microeconomics: Behavior, Institutions, and Evolution: Behavior, Institutions, and Evolution, Princeton University Press, 2009.

[30] R. N. Mantegna and H. E. Stanley, An Introduction to Econophysics: Correlations and Complexity in Finance, Cambridge University Press, Cambridge, UK, 2000.

[31] R. Dale, "Globalisation, knowledge economy and comparative education," Comparative Education, vol. 41, no. 2, pp. 117-149, 2005.

[32] K. Jaffe, "Extended inclusive fitness theory: synergy and assortment drives the evolutionary dynamics in biology and economics," SpringerPlus, vol. 5, no. 1, article 1092, 2016.

[33] K. Jaffe, M. Caicedo, M. Manzanares et al., "Productivity in Physical and Chemical Science Predicts the Future Economic Growth of Developing Countries Better than Other Popular Indices," PLoS ONE, vol. 8, no. 6, Article ID e66239, 2013. 


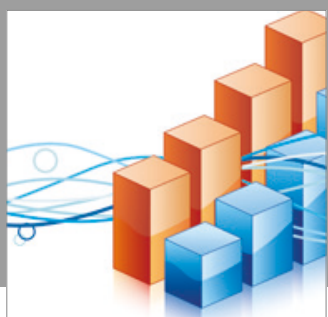

Advances in

Operations Research

vatersals

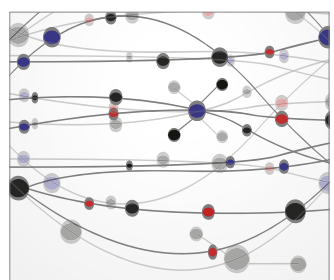

\section{The Scientific} World Journal
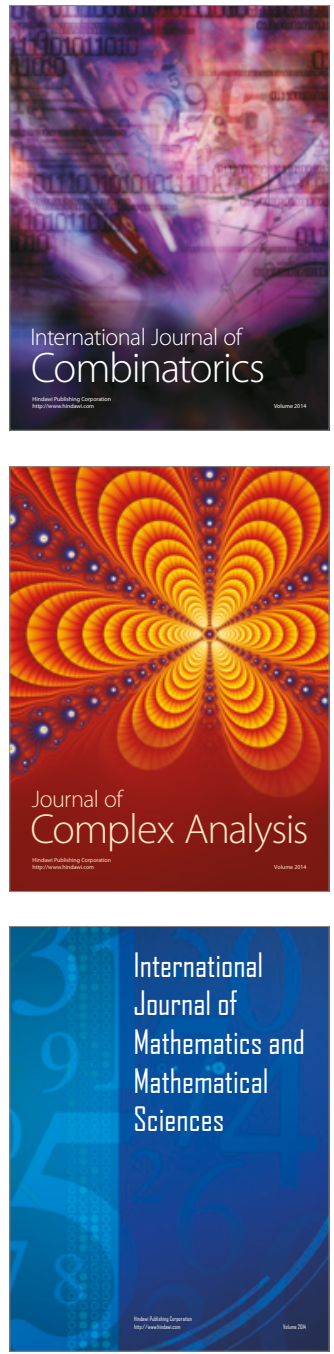
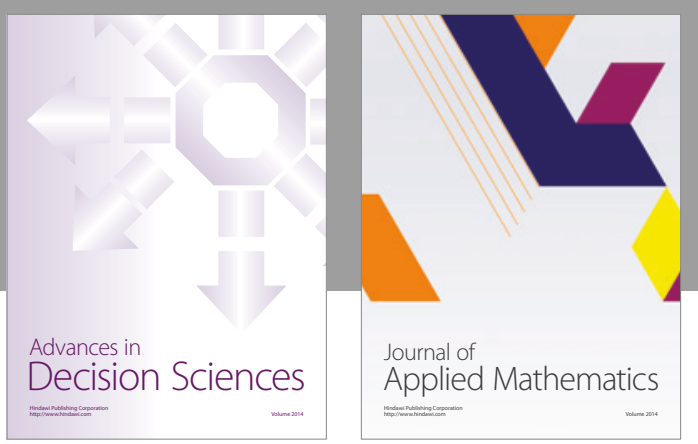

Algebra

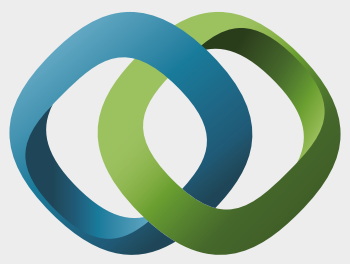

\section{Hindawi}

Submit your manuscripts at

https://www.hindawi.com
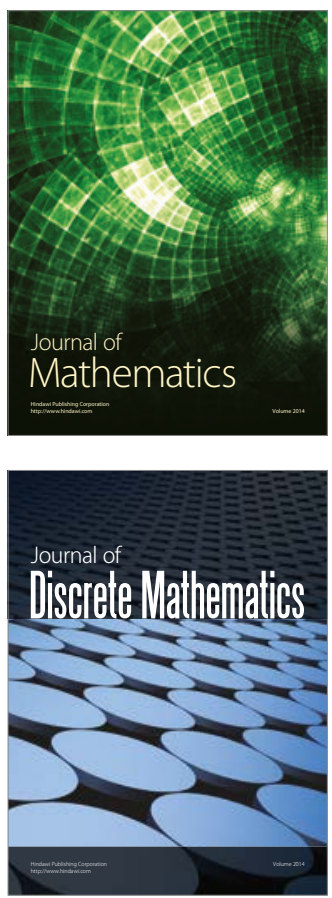

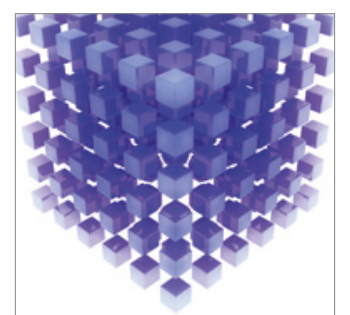

Mathematical Problems in Engineering
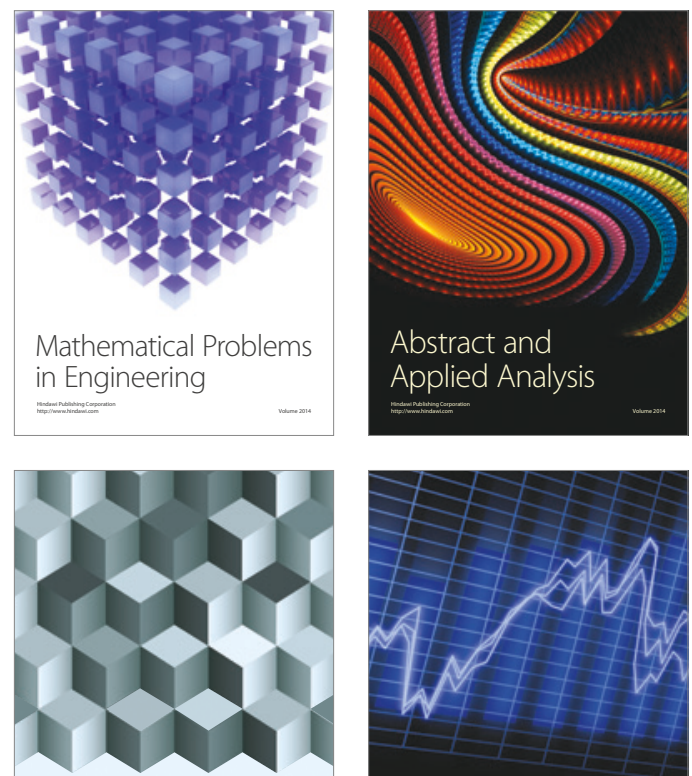

Journal of

Function Spaces

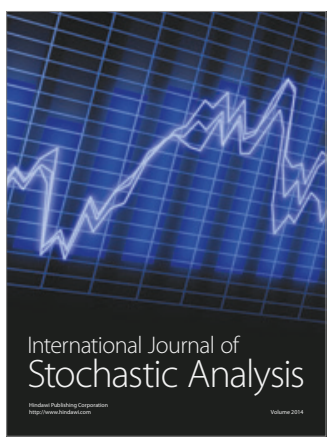

Probability and Statistics
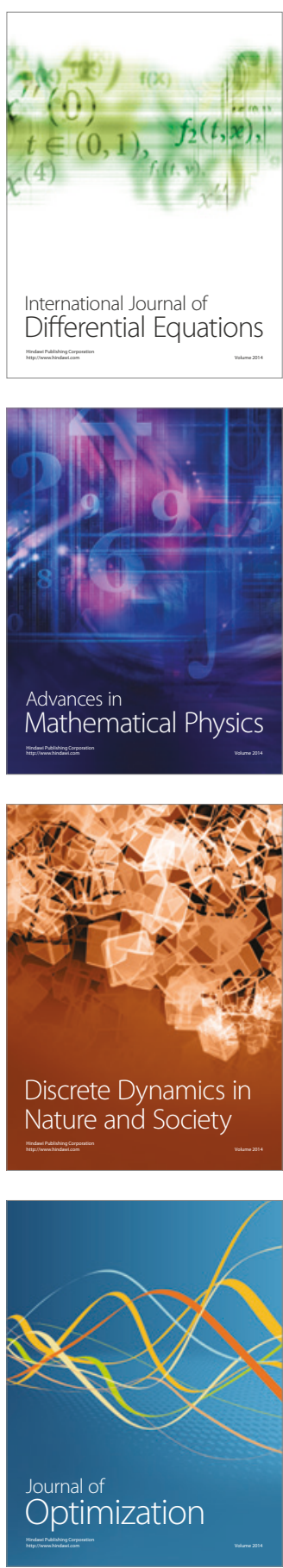